\title{
A Neural Model of Synaptic Plasticity Underlying Short-term and Long-term Habituation
}

\author{
DeLiang Wang* \\ Ohio State University
}

It has been demonstrated that short-term habituation may be caused by a decrease in release of presynaptic neurotransmitters and long-term habituation seems to be caused by morphological changes of presynaptic terminals. $A$ parsimonious model of short-term and long-term synaptic plasticity at the electrophysiological level is presented. This model consists of two interacting differential equations, one describing alterations of the synaptic weight and the other describing changes to the speed of recovery (forgetting). The latter exhibits an inverse S-shaped curve whose high value corresponds to fast recovery (short-term habituation) and low value corresponds to slow recovery (long-term habituation). The model has been tested on short-term and a set of long-term habituation data of prey-catching behavior in toads, spanning minutes to hours to several weeks.

Key Words: synaptic plasticity; short-term habituation; long-term habituation; modeling; toad

\section{Introduction}

It is a widely accepted view that memory has dichotomous forms: short-term memory (STM), which vanishes typically within minutes, and long-term memory (LTM), which can last for days, weeks or longer. Memory is believed to be caused by synaptic plasticity, and there is a good deal of evidence from many animal models--both vertebrates and invertebrates — suggesting that synapses can also undergo two forms of plasticity that, in general, parallel short-term and long-term behavioral changes (Thompson, 1986; Hawkins, Clark \& Kandel, 1987; Greenough \& Bailey, 1988; Dudai, 1989; Hawkins, Kandel \& Siegelbaum, 1993).

Habituation, defined as a decrease in the strength of a behavioral response to repeated stimulation, is probably the most elementary and ubiquitous type of learning (Thorpe, 1956; Thompson, 1986). Indeed, it occurs in all animals. An animal learns

\footnotetext{
* Laboratory for Artificial Intelligence Research, Department of Computer and Information Science and Center for Cognitive Science, Ohio State University, Columbus, OH 43210-1277; dwang@cis.ohio-state.edu
} 
by habituation to ignore the stimuli that occur repetitively but have no significance to the animal, and thus keeps alert to novel stimuli. Habituation therefore has critical survival values for the animal. Extensive studies have been undertaken to elucidate habituation behaviors and to understand their neurophysiological mechanisms. To distinguish habituation from other types of behavioral decrement (such as fatigue), Thompson and Spencer (1966) summarized a number of criteria of habituation, among which are (1) exponential decay of response strength with the number of stimulus presentations, (2) spontaneous recovery with rest, (3) more rapid and pronounced habituation with repeated series of habituation training, (4) generalization of habituation to similar stimuli, and (5) rapid recovery of the habituated response (dishabituation) on presentation of a different stimulus.

When studying habituation of prey-catching behavior in toads, Ewert (1967) observed that recovery of the response after habituation exhibits two phases: a shortterm one that lasts for a few minutes and a long-term one that lasts for at least 6 hours (see Ewert, 1984, for a review). Several years later, Carew, Pinsker, and Kandel (1972) reported long-term habituation of the siphon and gill withdrawal reflex in the marine mollusk Aplysia, which can last several weeks. Similar findings of both forms of habituation have been reported for the rat acoustic startle response (Leaton, 1976; Leaton \& Supple, 1991) and the crab escape response (Tomsic \& Maldonado, 1990). Interestingly, Koshland and his colleagues have recently observed both short-term and long-term habituation in single neurons in culture in response to repeated stimulation of either acetylcholine or adenosine triphosphate ( $\mathrm{McF}$ adden \& Koshland, 1990; Cheever \& Koshland, 1992).

Studies on synaptic mechanisms underlying behavioral habituation suggest that short-term habituation generally operates on presynaptic terminals as a result of reduced neurotransmitter release (Thompson, 1986; Greenough \& Bailey, 1988; Dudai, 1989; Hawkins, Kandel \& Siegelbaum, 1993). Long-term habituation, on the other hand, may be accompanied by structural changes of presynaptic terminals (Bailey \& Chen, 1983, 1988a; Greenough \& Bailey, 1988). As demonstrated in Aplysia, short-term and long-term habituation may share a common locus and certain aspects of a common mechanism (Hawkins, Clark \& Kandel, 1987). The idea that both STM and LTM are coded into the same sites (synapses) receives support from the studies of other forms of learning, such as sensitization and conditioning, and from research on other animal species (for a review, see Hawkins, Kandel \& Siegelbaum, 1993), including Hermissenda (Alkon et al., 1990), crayfish (Lnenicka, Atwood \& Marin, 1986), and frog (Herrera, Grinnell \& Wolowske, 1985).

Because it is well described behaviorally in a number of animal species and its cellular mechanisms have been revealed in simple systems, habituation has also been studied from the modeling perspective by a number of investigators. In particular, 
short-term habituation can be described quite well by a first-order differential equation that simulates the evolution of a synaptic weight, and such models in certain systems can fit the quantitative data very successfully (for details, see section 2 ). An important theoretical question is whether the two forms of habituation can be modeled simultaneously. Perhaps due to scarcity of systematic long-term habituation data or because LTM involves long simulations (just as in the experiments), little research has been devoted to this question. In one of few studies available, Ciaccia, Maio, and Vacca (1992) recently proposed a mathematical model for both short- and long-term classical conditioning. Their model, called the learning gate model, relies on biological data drawn on the study of Aplysia conditioning. This model introduces two connection weights for a single synapse, one representing long-term trace and another short-term trace. The overall synaptic efficacy is taken to be the product of the two (see also Gardner-Medwin, 1989). Several qualitative aspects of conditioning have been simulated by the learning gate model, including spontaneous recovery and second-order conditioning, but the model has not been tested on quantitative data of conditioning.

In this article, I will address the relationship between short- and long-term habituation and provide a unified model that can be used to explain both short- and long-term habituation data. To focus our investigation, my colleagues and I chose toad visual habituation as the prototype. There are a number of reasons for this choice. First, as mentioned earlier, there exist systematic behavioral data on both short- and long-term habituation. Second, the toad visual system is relatively simple compared to other higher vertebrates and has been a subject of extensive biological study (for reviews, see Llinás \& Precht, 1976; Ingle, 1983; Ewert, 1984; Ewert et al., 1992). Third, toad visual habituation has served as a model of our computational studies in the past. In particular, we have simulated short-term habituation of visually induced prey-catching behavior (Wang \& Arbib, 1992), which enables the animal to discriminate similar visual stimuli (Wang \& Arbib, 1991a). Ultimately, we will argue that the model proposed here is not limited to toad visual habituation and that the basic principles may be applicable to modeling other kinds of habituation as well.

\section{A Model of Synaptic Plasticity}

Once the intensity of a behavior (an animal's response) can be quantitatively measured, habituation can often be quantified by changes in the intensity of the behavior. It is thus interesting to explain the observed quantitative data by a computational or mathematical model. The general idea behind this type of modeling is to view habituation as an isolated process of synaptic plasticity and to link modification of model synapses directly to behavioral changes (see Stanley, 1976, for a typical treatment). 
In simple systems, this type of model may be readily applied to explain cellular processes of habituation. In more complex systems, such as prey-catching behavior in toads, which involves many neurons in several visual nuclei, this simple way of linking behavioral decrement to synaptic plasticity appears insufficient and perhaps simplistic. The justification behind this approach for complex behaviors, we believe, is that as long as synaptic weights that undergo habituating modification eventually have a multiplicative role in determining the behavioral outcome, synaptic plasticity directly corresponds to behavioral changes. This condition holds for many neural models of adaptive behavior, including our model, as we will see later. In this sense, the study of habituation as an isolated process can contribute to the understanding of neural mechanisms underlying behavioral habituation.

Mathematically, the decrease of synaptic efficacy $\gamma$ that gives rise to habituation is mostly modeled by a first-order differential equation (see, among others, Stanley, 1976; Gingrich \& Byrne, 1985; Lara \& Arbib, 1985; Gluck \& Thompson, 1987; Ögmen \& Moussa, 1993):

$$
\tau \frac{d y(t)}{d t}=\alpha\left[\gamma_{0}-\gamma(t)\right]-S(t)
$$

where $y_{0}$ is the initial value of $y ; S(t)$ represents external stimulation to the synapse; $\tau$, the time constant, governs the rate of habituation; and $\alpha$ regulates the rate of recovery. This linear differential equation exhibits both exponential decrease with repeated stimulation and exponential recovery when the stimulus is withdrawn, which can be easily seen from the following analysis. Let $S(t)$ be a positive constant, denoted as $S$, when the stimulus is applied. With the assumption that $\gamma(0)=\gamma_{0}$, the solution for Equation 1 is

$$
y(t)=\gamma_{0}-\frac{S}{\alpha}[1-\operatorname{Exp}(-\alpha t / \tau)]
$$

thus showing exponential decay with time. Here Exp symbolizes the exponential function. When the stimulus is withdrawn-that is, $S(t)=0$ - the solution of Equation 1 becomes $\gamma(t)=\gamma_{0}-\left(\gamma_{0}-\gamma_{1}\right) \operatorname{Exp}(-\alpha t / \tau)$, where $\gamma_{1}$ is the value of $\gamma$ at the time of stimulus withdrawal. Thus, the weight $y$ exponentially recovers to its initial value $\gamma_{0}$.

Although Equation 1 successfully describes exponential decay and recovery, the two basic properties of habituation (Thompson \& Spencer, 1966; Ewert, 1984; Cheever \& Koshland, 1992), it can model only short-term habituation (more explanations follow). When simulating Aplysia's habituation (Wang \& Hsu, 1990) and toad's stimulus-specific habituation (Wang $\&$ Arbib, 1992), we proposed an idea to incorporate both short- and long-term habituation by using an inverse S-shaped curve (Fig. 1) to describe two forms of memory. Such a curve can be roughly di- 


\section{Figure 1}

Ten $z(t)$ curves of Equation 4 with different parameter values. $t_{11}=5$, $6,7,8,9$, respectively, and $\gamma=0.5$ for the thick curves and 1.0 for the thin curves (see Wang \& Arbib, 1992, for a similar graph).

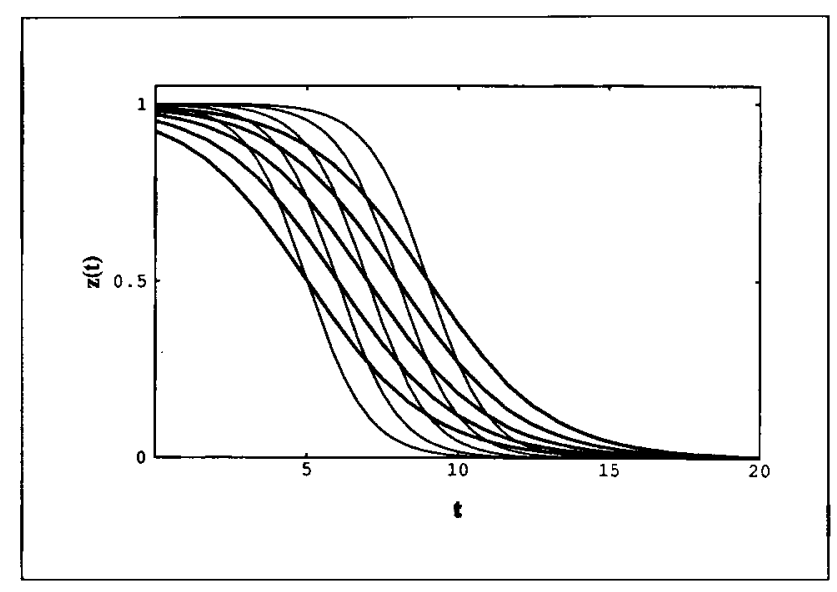

vided into three stages: a high-value stage, a low-value stage, and a middle transition stage around the inflection point, during which a rapid transition from a high value to a low value occurs. Linking to STM and LTM, the high-value and low-value stages correspond to the two forms of memory. This idea is now adapted to a neural model of the following form:

$$
\begin{aligned}
& \tau \frac{d y(t)}{d t}=\alpha z(t)\left[\gamma_{0}-\gamma(t)\right]-\beta \gamma(t) S(t) \\
& \frac{d z(t)}{d t}=\gamma z(t)[z(t)-1] S(t)
\end{aligned}
$$

where $\gamma, \gamma_{0}, \tau$, and $\alpha$ are as described for Equation 1. The first term in Equation 3a regulates recovery to $y_{0}$, and its rate is controlled by variable $z(t)$, defined in Equation $3 \mathrm{~b}$. The second term in Equation 3a regulates habituation, with $\beta$ controlling the effectiveness of the presynaptic stimulus $S(t)$, which is multiplied by $\gamma(t)$ to form the activity-gated input (see also Ögmen \& Moussa, 1993), as opposed to direct input in Equation 1. The intuition behind the activity-gated input is that a stimulus reduces the synaptic weight more rapidly in an early stage when the weight is relatively large. In addition, Equation 3a naturally reflects the constraint that the weight $\gamma(t)$ never goes below 0 . This is because the smaller (but still positive) the value of $y$, the less the second term (negative one) on the right-hand side of Equation 3a reduces the value of $\gamma$. Eventually, due to the first term of Equation 3a, an equilibrium is reached before $\gamma$ reduces to 0 . On the other hand, if $S$ is too large compared to $\alpha$ in Equation 1, $\gamma$ can reduce to a negative value, as is clearly shown in its solution of Equation 2. 
If there is no external stimulation $[S(t)$ is set to 0$], z(t)$ does not change its value. To study the behavior of Equation $3 \mathrm{~b}$, let us assume without loss of generality that there is a constant unity stimulus. Then Equation $3 b$ becomes $d z(t) / d t=\gamma z(t)[z(t)-1]$. With the initial condition $t=t_{0}, z(t)=0.5$, the solution to the equation is

$$
z(t)=\frac{1}{1+\operatorname{Exp}\left[\gamma\left(t-t_{0}\right)\right]},
$$

which is a typical inverse $\mathrm{S}$-shaped curve with the inflection point at $t=t_{0}$. The transition speed from the high-value stage to the low-value stage is controlled by the slope at the inflection point, which equals $-\gamma / 4$. The overall speed of the decrease of $z(t)$ is controlled by $t_{0}$, and the larger is $t_{0}$, the slower is the speed of decrease of $z(t)$. Figure 1 shows two groups of $z$ curves with different values of $\gamma$ and $t_{0}$. It is clear from Equation 3a that the effect of $z(t)$ on $\gamma(t)$ is to control the rate of recovery. When $z(t)$ is in the high-value stage, recovery is relatively fast; when $z(t)$ is in the low-value stage, recovery is relatively slow. These two stages of recovery are used to model two forms of memory, STM and LTM. It should be clear that $z(t)$ evolves much slower than $y(t)$.

The idea behind the basic assumption of the inverse $\mathbf{S}$-shaped curve is the following: As mentioned in section 1, long-term habituation appears to be caused by structural changes of presynaptic terminals as a result of repeated presynaptic stimulation. Because of the behavioral dichotomy of STM and LTM, as opposed to a smooth transition from STM to LTM, there must be a nonlinear relation between the amount of the structural changes and the amount of stimulation. An inverse Sshaped curve (known as a logistic function in applied mathematics) seems to provide a minimum model that captures this type of nonlinearity with two global states (STM and LTM) and yet accommodates a dimension of flexibility. Figure 1 illustrates how the parameters of Equation $3 \mathrm{~b}$ modify the detailed shape of a curve. This kind of flexibility is absent from simpler formulations of nonlinearity, say binary $(0 / 1)$ or polynomial functions.

\section{Computer Simulation of Short-term and Long-term Habituation in Toads}

Visually induced prey-catching orienting behavior in toads and frogs has long been known to be habituatable. In common toads, this habituation exhibits the same characteristics as summarized by Thompson and Spencer (1966). Ewert and his colleagues have conducted extensive behavioral experiments on this type of visual habituation in toads (Ewert, 1967, 1970, 1984; Ewert \& Kehl, 1978), and their systematic data form an ideal test bed for quantitative habituation models at both time scales. Short-term habituation, particularly the data of Ewert and Kehl (1978), 


\section{Figure 2}

Visual pathway underlying habituation of prey-catching behavior in toads. Arrowheads indicate the direction of neural fiber projection, and the dashed arrowhead indicates an indirect projection. OT, optic tectum; AT, anterior thalamus; MP, medial pallium.

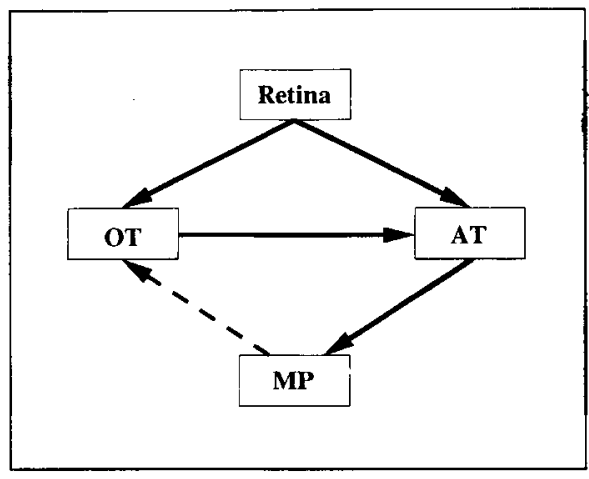

has been previously modeled by Lara and Arbib (1985) and Wang and Arbib (1992). This article is concerned mainly with modeling long-term habituation.

Before giving simulation details, I will briefly describe the context of the following simulations, in the hope of convincing the reader that linkage between the synaptic plasticity model of Equation 3 and later behavioral modeling is justified. Figure 2 provides a diagram of the visual structures involved in visual pattern discrimination in toads. The retina, after processing the stimuli of optic flow, sends its outputs to both the optic tectum (OT) and the anterior thalamus (AT). OT is a major neural structure for amphibian visual processing and, as one of the several efferent pathways, OT projects to AT, which further projects ascendingly to the medial pallium (MP) in the telencephalon. MP descendingly connects to OT indirectly, possibly via the preoptic region (PO) or the hypothalamus (HYP), thus forming a neural loop. This loop (tectum $\rightarrow A T \rightarrow M P \rightarrow P O / H Y P \rightarrow$ tectum $)$ is what Ewert (1987) called the modulatory loop, which is supposed to modulate via learning visual prey-catching behavior. In particular, ample evidence suggests that MP, the homolog of mammalian hippocampus (Herrick, 1933), is the neural structure underlying various learning types including habituation (for review of the relevant data, see Ewert, 1987; Ewert et al., 1992; Wang \& Arbib, 1992). Based on these anatomical as well as neurophysiological data, Wang and Arbib (1991a,b) have previously modeled the neural structures of retina, OT, and AT in toads and frogs (see Figure 2). To address shortterm habituation, we also modeled an MP column, corresponding to a specific visual location (Wang \& Arbib, 1992). From the habituation perspective, the MP model of Wang and Arbib basically satisfies the condition that the habituating synaptic weights have a multiplicative role in determining the overall behavioral output. Because, in the following simulations, we are mainly concerned with the changes of a response with respect to time, directly simulating Equation 3 is equivalent to presenting a visual stimulus and measuring the changes of its response after several steps of retinal, 


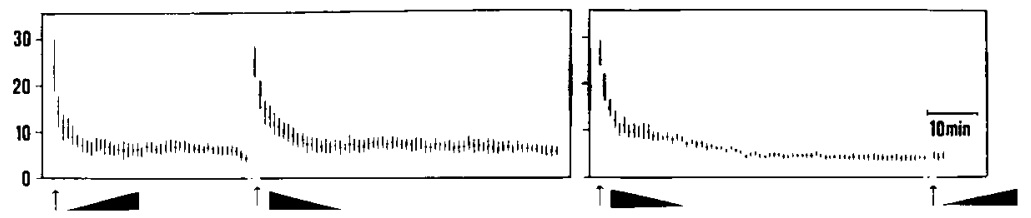

b

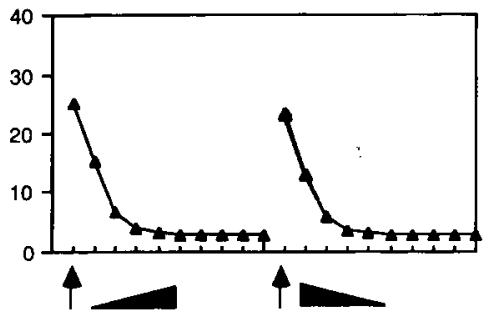

6

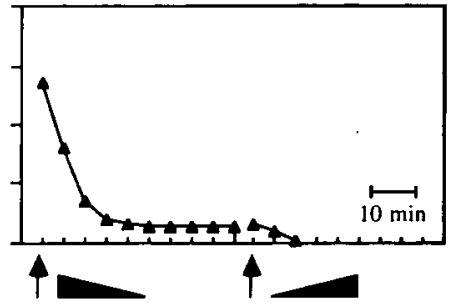

\section{Figure 3}

Simulation of single-session habituation and dishabituation of prey-catching orienting response in toads. (a) The experimental results obtained by Ewert and Kehl (1978) concerning two different visual stimuli. In the left frame, the left-pointing triangle was presented first and immediately after its habituation, the right-pointing triangle was presented and habituated. The right frame reflects the reverse order of presentation. (b, c) The corresponding simulation results. Each data point in (b) and (c) is measured as the relative value of the initial response of a frame that is scaled to the same value in (a). The parameter values are: $\gamma_{0}=1.0 ; \tau=200 ; \alpha=3.2 ; \beta=24 ; \gamma=0.1$. As is clear from Equation 4 , there is a one-to-one correspondence between $z(0)$, the initial value of $z$, and $t_{0} ; z(0)=0.9999$ in the simulation. $S(t)$ is set to 1 if the external stimulation is on, or 0 otherwise.

tectal, and thalamic processing. Of course, due to its computational advantages, the former method was adopted in our simulations of long-term habituation that follow.

We tested the synaptic plasticity model (Equation 3) against the single-session, short-term habituation data of Ewert and Kehl (1978). This simulation also helps constrain the parameters of Equation 3. Figure 3a displays the data of Ewert and Kehl concerning two stimuli, the mirror images of a triangle. After a single session of habituation to the left-pointing triangle, the toads immediately responded to the presentation of the right-pointing triangle and experienced similar habituation when the latter stimulus was presented repeatedly. However, following habituation to the right-pointing triangle, the animals hardly responded to a later presentation of the left-pointing triangle. To model habituation to a specific stimulus, Equation 3 was embedded in the MP model (Wang \& Arbib, 1992) that performs shape dis- 
crimination. The details of shape discrimination (see Wang \& Ewert, 1992) are not important here, as behavioral changes are all that matters for habituation. In the simulation, each stimulus was presented for 60 minutes and then switched to another stimulus, which also was presented for 60 minutes. For visualization purposes, we present only ten data items for each session, each corresponding to a 6-minute interval. In all the simulations presented in this article, time was measured as follows: A basic discretization step 0.05 corresponds to 1 second. Figure $3 \mathrm{~b}$ and $\mathrm{c}$ present the corresponding simulation results. In Figure $3 \mathrm{~b}$, the left-pointing triangle was presented first, and the model response was habituated after repeated presentation of the stimulus for 60 minutes. Immediately afterward, the right-pointing triangle was presented, and it triggered a new response that also was habituated after repeated presentation. The reverse order of presentation was studied in Figure $3 c$, and apparently no new response was demonstrated when the left-pointing triangle was presented after habituation to the right-pointing triangle.

From the comparison between Figure $3 \mathrm{~b}$ and $\mathrm{c}$ and Figure $3 \mathrm{a}$, it can be concluded that the habituation model can reproduce the experimental data. In particular, the detailed time course of habituation in the simulation compares well with the experimental data. With the same parameter values (see the caption of Figure 3), the same good results were also found in other simulations corresponding to the Ewert and Kehl experiments.

The quantitative data of Ewert and Kehl (1978) could also be modeled with just an STM model (Lara \& Arbib, 1985; Wang \& Arbib, 1992), as habituation tests were conducted within only one session. Thus, it is not particularly demanding to obtain the results in Figure 3. On the other hand, rather early observations by Ewert $(1967,1970,1984)$ are surprisingly suitable for long-term habituation tests, Ewert's habituation experiments being conducted in series of training sessions separated by recovery pauses. During each training session, toads continuously oriented to a moving prey dummy until their responses were below a criterion value. The next session started after a recovery pause during which previously acquired habituation was partly forgotten. Figure 5a summarizes the experimental results. Apparently, the longer an intersession pause, the more activity will be released during the following session. The log scale of the ordinate of Figure $5 \mathrm{a}$ indicates that the overall response in a training session approximately drops exponentially with the number of sessions (Ewert, 1984). This exponential decay appears to be in accordance with the exponential decay of short-term habituation (see Fig. 3), but this is not the case, as is shown by the following simple analysis: After a constant recovery pause $T$, during which $S(t)=0$, we have from Equation 3a:

$$
y=y_{0}-\left(\gamma_{0}-y_{m}\right) \operatorname{Exp}(-\alpha z T / \tau)
$$




\section{Figure 4}

Simulation of a habituation series with a 5-minute intersession pause. In the simulation, the instantaneous response of the model is assumed to be proportional to the synaptic weight, $\gamma(t)$. The log scale is used for the ordinate. As in the experiments, a simulated training session stops whenever $\gamma(t)$ falls below $y_{m}$ (Equation 5), and $\gamma_{m}=0.015$. The rest of the parameter values are the same as for Figure 3.

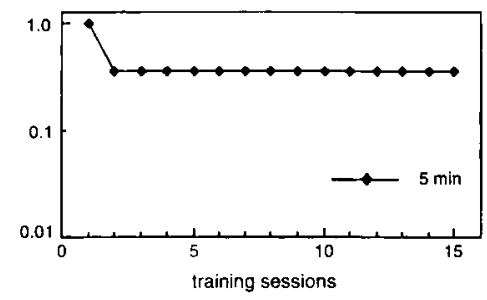

where $\gamma_{m}$ is the weight at which experiment tests are stopped, corresponding to the criterion value of habituation. If only STM is concerned (i.e., $z$ is set to 1), it is clear from Equation 5 that the synaptic weight will recover to a constant level regardless of prior training sessions. In other words, after an initial decay, the overall activity elicited during each session is a constant. This is clearly shown in Figure 4 which presents a simulation of a habituation series of 15 sessions with a 5 -minute pause. In this simulation, $z$ is set to 1 . As in the experimental data of Figure 5a, each data point in Figure 4 represents the normalized value of the overall model response over one session. Figure 4 evidently contradicts the experimental data of Figure 5a. Notice that this contradiction is not a result of the detailed definition of Equation 3a. The same phenomenon occurs when Equation 3a is replaced by commonly used Equation 1. Therefore, a short-term habituation model is not able to explain the data, and LTM must be taken into consideration. This is expected since the experiments involved long training series.

The challenge for our model becomes whether Equation 3 can be used to reproduce the quantitative data of Figure 5a that demand a long-term habituation model. A further challenge is whether Equation 3 can achieve this with the same set of parameters as in Figure 3, which were tuned to reproduce successfully the short-term (single-session) habituation data. We conducted extensive simulations to produce corresponding model outputs to Figure 5a. Fortunately, during a recovery pause, the value of $z(t)$ does not change because $S(t)=0$, and thus the value of $y(t)$ can be analytically solved. Even with analytical solutions, simulation of each series of habituation sessions is computationally intense because each session lasted for tens of minutes. Also, in Figure 5a, there are three series with many data points to simulate. This is particularly challenging to model with the few parameters in Equation 3, most of which were already tightly constrained in simulating quantitative data of short-term habituation. Figure $5 \mathrm{~b}$ presents the simulation results using the same parameters as in Figure 3. Each series in the simulation consisted of 15 training 


\section{Figure 5}

Data and simulations of successive habituation in toads. (a) Habituation effects in successive training sessions separated by constant recovery pauses of 1 minute, 5 minutes, and 24 hours. Each data point represents the normalized value of the cumulative number of orienting turns that the animal released in response to a continuously moving prey dummy during one habituation session. (Redrawn from Ewert, 1984.) (b) The corresponding simulation results. The parameter values are the same as for Figure 3 except $y_{m}=0.015$ as in Figure 4. (c) The time course of $\gamma$ and $z$ in the entire process of 15 training and recovery sessions with a 5-minute intersession pause. Each pause is accompanied in the figure by an increase segment in $\gamma$ (spontaneous recovery) and a constant segment in $z$. a

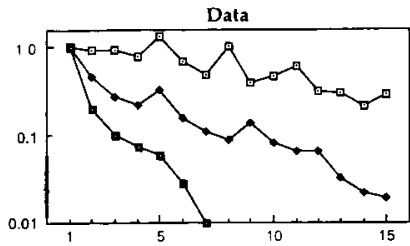

b
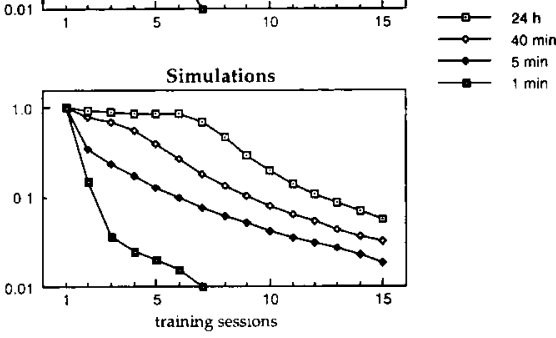

c
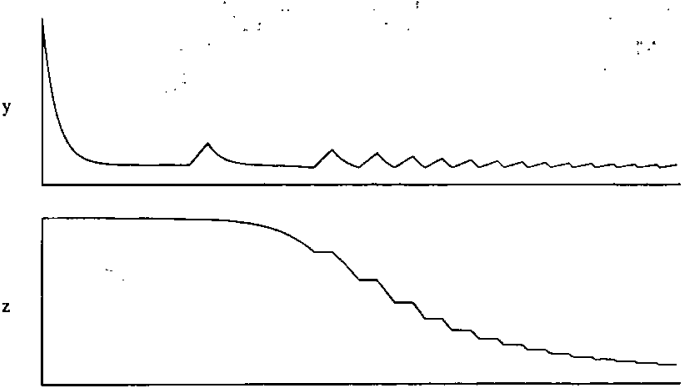

sessions, and 1-minute, 5-minute, and 24-hour intersession pauses were used. Other details of the simulation are consistent with those in Figures 3 and 4 . Compared to Figure $5 \mathrm{a}$, the simulation results are quantitatively similar to the experimental data. Figure $5 c$ displays a typical time course of $\gamma(t)$ and $z(t)$ with a 5-minute pause.

The decrease of the overall response in later training sessions is due to two factors: the decrease of the response amplitude, which is proportional to the synaptic weight, and the decrease of the duration of a training session. The interaction of these two factors gives rise to the two linear trends, particularly evident in the 1 -minute and the 24-hour curves of Figure 5b. Different linear trends also seem to occur in the data curves of Figure 5a; this is clearly evident on the 1-minute curve. For the 24-hour case, it seems that the recovery (forgetting) occurs more slowly in the model than in real animals, as is particularly evident for the second half of the curve in Figure $5 \mathrm{~b}$. 
This suggests that the switch from one linear trend to another in the model should occur more smoothly, a topic of future research. Also, the oscillatory fluctuations of the curves in Figure 5a seem to call for additional mechanisms (Ewert, 1984).

To compare model predictions and other sets of behavioral data not included in original modeling, we also conducted a habituation series with a 40-minute pause after the model was completely fixed. This model prediction curve is included in Figure $5 \mathrm{~b}$. It matches well the preliminary observations of a corresponding habituation series of toad's prey-catching behavior by Ewert (1967). A more rigorous test of this prediction, however, must await future experiments in which more toads should be used.

\section{Discussion}

The main point of this article is a demonstration that STM and LTM processes may be quantitatively modeled as a result of a common dynamical process. A sharp decrease in the transition stage of $z$ underlies the transfer from an STM process to an LTM process. The model does not imply that STM and LTM physically share the same neural mechanisms. Our modeling, however, suggests that short-term and long-term processes are not independent. As mentioned earlier, short-term habituation involves alterations in neurotransmitter release, whereas long-term habituation seems to involve structural changes such as the number of presynaptic terminals (varicosities) and the number and size of active zones (Bailey \& Chen, 1988a, 1988b). It is interesting to note that similar mechanisms also underlie short-term and long-term synaptic plasticity at the frog neuromuscular junction (Magleby \& Zengel, 1982; Herrera, Grinnell \& Wolowske, 1985). Based on these observations, we suggest in gross terms that Equation 3a provides a description of changes in neurotransmitter release and Equation $3 \mathrm{~b}$ provides a description of morphological changes that accompany long-term habituation. As can be seen from Equation 3a, morphological changes then affect the process of neurotransmitter release by changing its recovery process. Thus, the two forms of synaptic plasticity can be unified by the interacting equations.

We have also tried to incorporate other possible descriptions for the long-term variable $z(t)$ of Equation 3. In particular, we tested a similar description of $\gamma(t)$ for $z(t)$ so that $z(t)$ also exhibits an exponential decay, as the exponential decay in Figure 5a seems to favor such a choice. However, the results of other forms of $z(t)$, including the exponential decay description, are not nearly as comparable with Figure $5 \mathrm{a}$ as is Equation $3 \mathrm{~b}$.

Synaptic plasticity in general is underlain by molecular changes that take place in presynaptic terminals on repeated stimulation from presynaptic neurons. Clearly, 
our model has not specifically addressed molecular and ionic channel modifications accompanying habituation, though other models have attempted to deal with these (see, for example, Magleby \& Zengel, 1982; Gingrich \& Byrne, 1985). Rather, we have attempted to provide phenomenological descriptions of how synaptic weights are modified by stimulation. Such a style of habituation modeling can be found extensively in the literature (see, among others, Stanley, 1976; Lara \& Arbib, 1985; Gluck \& Thompson, 1987; Ögmen \& Moussa, 1993). The distinction between these two kinds of modeling resembles that between the detailed Hodgkin-Huxley equations (Hodgkin \& Huxley, 1952) for a single neuron's action potential generation and the more abstract models of FitzHugh (1961) and Nagumo, Arimoto, and Yoshizawa (1962) for generating neuronal impulses. The obvious advantage of more abstract models lies in their analytical simplicity and computational efficiency.

Of course, these types of equation cannot generate predictions about how molecular processes operate during habituation. However, quantitatively tested abstract models can generate useful predictions about animal behavior. For instance, our model of toad's visual discrimination produces a number of precise behavioral predictions (Wang \& Arbib, 1991a), which helped to design a set of experiments to test and actually validate some of the predictions (Wang \& Ewert, 1992). The 40minute curve in Figure $5 \mathrm{~b}$ is a behaviorally testable prediction about our quantitative description of synaptic plasticity. In addition to behavioral implications, Equation 3 suggests that presynaptic stimulation affects consolidation of long-term habituation following the inverse $\mathrm{S}$-shaped curve of Equation 4, and the long-term trace affects short-term habituation by slowing down its recovery time scale (the first term of the right-hand side of Equation 3a).

Although the learning gate model of Ciaccia, Maio, and Vacca (1992) addresses conditioning while ours models habituation, some comparisons may be drawn about how STM relates to LTM in these two models. The learning gate model introduces two different weights, one short-term and another long-term, and assumes that the overall weight is a product of the two. The same assumption was used earlier by Gardner-Medwin (1989), who proposed an associative memory model using both short-term and long-term synaptic modification. In Equation 3, we use only one synaptic weight with the introduction of $z$, which controls both time scales of forgetting. Another difference is that our model uses an inverse $S$-shaped curve to describe the transition from STM to LTM, whereas the learning gate model uses an exponential function similar to Equation 1 to describe LTM but with a much slower time scale than the one for STM.

Although the model is tested mainly on the habituation data from toads, application of the model is not necessarily restricted to anuran amphibians. The basic properties of Equation 3 are consistent with the general parametric features of 


\section{Figure 6}

Time course of long-term habituation.

(a) Habituation of the siphon withdrawal reflex in Aplysia. Each data point is the accumulated score of the median response from ten training trials within a day. The response is measured as the duration of siphon withdrawal. Following the first 4 days of training, retention was tested on days 5,12 , and 26 .

(Redrawn from Carew, Pinsker \& Kandel, 1972.)

(b) Habituation of the acoustic startle response in the rat. Each data point is the mean startle amplitude (in millimeters) to a tone stimulus. One training session was conducted daily. (Redrawn from Leaton, 1976.) a

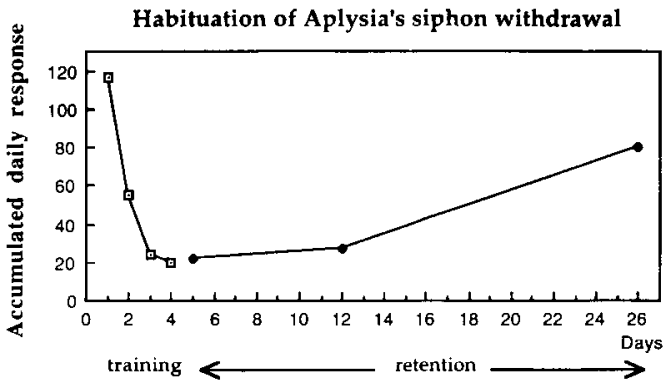

b

Habituation of rat acoustic startle

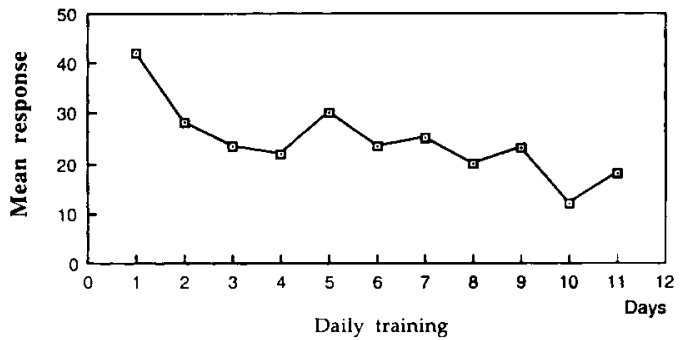

habituation summarized by Thompson and Spencer (1966) (see section 1). As shown by the $y$ curve in Figure $5 c$, this model exhibits exponential decay of response strength and spontaneous (also exponential) recovery with rest. Figure $5 \mathrm{~b}$ clearly shows that habituation occurs more rapidly and strongly with a series of training sessions. Generalization and dishabituation generally involve the features of stimuli but, as shown in Figure 3, this habituation model, together with a stimulus discrimination system, can readily demonstrate generalization and dishabituation.

More specifically, the exponential decay of the overall response after habituation series, as shown in Figure $5 \mathrm{a}$, seems to be a general property of long-term habituation across different animal species. Figure 6 displays the systematic data of two other habituation series, obtained from the studies of Aplysia's siphon withdrawal reflex (Carew, Pinsker \& Kandel, 1972) and the rat acoustic startle response (Leaton, 1976). Each point in Figure 6a shows the accumulated score of ten habituation trials conducted during 1 day. Here we are mainly interested in habituation exhibited in the first 4 days, which can be compared with the data of Figure 5a by assuming an approximate 24-hour intersession pause. Each point in Figure $6 \mathrm{~b}$ represents the 
response to a single habituation trial conducted daily. This decrement pattern, however, is similar to the later experiments in which each daily session consists of six trials (Leaton \& Supple, 1991). The behavioral decrements in these two studies are displayed in the linear scale, as opposed to the log scale of Figure 5a. Thus, the exponential trend of the decrement following daily habituation training exhibited in both graphs appears consistent with Figure 5a. More careful inspection also shows that the detailed decrement curves during each daily session both in Aplysia (Carew, Pinsker \& Kandel, 1972) and in the rat (Leaton \& Supple, 1991) are qualitatively similar to the $y$ curve of Figure $5 c$. Therefore, we conclude that the basic properties of the long-term habituation data in toads may be exhibited by other animal species as well.

It is not unreasonable to suspect that the laws of synaptic plasticity at the basic level may be universal to all types of learning. For example, a reverse but, by nature, similar process has been demonstrated in sensitization (Castellucci \& Kandel, 1976; Bailey \& Chen, 1988b; Hawkins, Kandel \& Siegelbaum, 1993), and the two forms of conditioning seem to interact with each other in a way similar to habituation (Carew, Walters \& Kandel, 1981; Buonomano \& Byrne, 1990). In the future, we plan to use the same idea to address LTM data of sensitization and conditioning, and to see to what extent the idea can be utilized to simulate LTM in general.

Our model provides a parsimonious description of the fundamental processes of short-term and long-term plasticity at the synaptic level, as distinguished from more detailed modeling of ionic channels (Magleby \& Zengel, 1982; Byrne, 1982). From a computational perspective, a simpler description at a basic level may result in significant gains of computational power at the network (systems) level (see Kohonen, 1989, and Wang \& Arbib, 1992, for examples of habituation at the network level). Admittedly, there are many issues, such as interactions between different ionic channels, that are overlooked in the phenomenological description of synaptic weight modification. Future research will address the question of how to relate the phenomenological descriptions of synaptic plasticity and the detailed molecular processes accompanying it. But we believe that simple but fairly accurate quantitative descriptions of synaptic plasticity at the cellular level merit their own studies. For instance, Equation 3 may have to be changed in light of new emerging data or may simply give way to more theoretically elegant descriptions. Lastly, our analysis shows that quantitative data from series of training sessions, which are relatively easy to obtain, may well serve to verify the type of quantitative LTM model proposed in this article. We hope that experimental tests of such models and further refinement of modeling will enhance our understanding of the relationship between STM and LTM.

\section{Acknowledgment}

I thank Dr. B. Givens for critically reading the manuscript. I also thank the anonymous referees and my editor for their critical comments and constructive suggestions. 
Preparation of this article was supported in part by National Science Foundation grant no. IRI-9211419 and Office of Naval Research grant no. N00014-93-1-0335.

\section{References}

Alkon, D. L., Ikeno, H., Dworkin, J., McPhie, D. L., Olds, J. L., Lederhendler, I., Matzel, L., Schreurs, B. G., Kuzirian, A., Collin, C., and Yamoah, E. (1990). Contraction of neuronal branching volume: An anatomic correlate of Pavlovian conditioning. Proceedings of the National Academy of Sciences of the United States of America, 87, 1611-1614.

Bailey, C. H., \& Chen, M. C. (1983). Morphological basis of long-term habituation and sensitization in Aplysia. Science, 220, 91-93.

Bailey, C. H., \& Chen, M. C. (1988a). Long-term memory in Aplysia modulates the total number of varicosities of single identified sensory neurons. Proceedings of the National Academy of Sciences of the United States of America, 85, 2373-2377.

Bailey, C. H., \& Chen, M. C. (1988b). Long-term sensitization in Aplysia increases the number of presynaptic contacts onto the identified gill motor neuron L7. Proceedings of the National Academy of Sciences of the United States of America, 85, 9356-9359.

Buonomano, D. V., \& Byrne, J. H. (1990). Long-term synaptic changes produced by a cellular analog of classical conditioning in Aplysia. Science, 249, 420-423.

Byrne, J. J. (1982). Analysis of the synaptic depression contributing to habituation of gill-withdrawal reflex in Aplysia californica. Journal of Neurophysiology, 48, 431-438.

Carew, T. J., Pinsker, H. M., \& Kandel, E. R. (1972). Long-term habituation of a defensive withdrawal reflex in Aplysia. Science, 175, 451-454.

Carew, T. J., Walters, E. T., \& Kandel, E. R. (1981). Associative learning in Aplysia: Cellular correlates supporting a conditioned fear hypothesis. Science, 211, 501-504.

Castellucci, V. F., \& Kandel, E. R. (1976). Presynaptic facilitation as a mechanism for behavioral sensitization in Aplysia. Science, 194, 1176-1178.

Cheever, L., \& Koshland, D. E., Jr. (1992). Retention of habituation in PC12 cells. Proceedings of the National Academy of Sciences of the United States of America, 89, 10084-10088.

Ciaccia, P., Maio, D., \& Vacca, G. P. (1992). An analytical short- and long-term memory model of presynaptic plasticity. Biological Cybernetics, 67, 335-345.

Dudai, Y. (1989). The neurobiology of memory: Concepts, findings, trends. New York: Oxford University Press.

Ewert, J.-P. (1967). Untersuchungen über die Anteile zentralnervöser Aktionen an der taxisspezifischen Ermüdung beim Beutefang der Erdkröte (Bufo bufo L.). Zeitschrift für Vergleichende Physiologie, 57, 263-298.

Ewert, J.-P. (1970). Neural mechanisms of prey-catching and avoidance behavior in the toad (Bufo bufo L.). Brain, Behavior and Evolution, 3, 36-56.

Ewert, J.-P. (1984). Tectal mechanisms that underlie prey-catching and avoidance behaviors in toads. In H. Vanegas (Ed)., Comparative neurology of the optic tectum. New York: Plenum.

Ewert, J.-P. (1987). Neuroethology: Toward a functional analysis of stimulus-response mediating and modulating neural circuitries. In P. Ellen and C. Thinus-Blonc 
(Eds.), Cognitive processes and spatial orientation in animal and man (Part 1). Dordrecht: Martinus Nijhoff.

Ewert, J.-P., Beneke, T. W., Buxbaum-Conradi, H., Dinges, A. W., Fingerling, S., Glagow, M., Schürg-Pfeiffer, E., \& Schwippert, W. W. (1992). Adapted and adaptive properties in neural networks for visual pattern discrimination: A neurobiological analysis toward neural engineering. Adaptive Behavior, 1, 123-154.

Ewert, J.-P., \& Kehl, W. (1978). Configurational prey-selection by individual experience in the toad Bufo bufo. Journal of Comparative Physiology, A, 126, 105-114.

FitzHugh, R. (1961). Impulses and physiological states in models of nerve membrane. Biophysical Journal, 1, 445-466.

Gardner-Medwin, A. R. (1989). Doubly modifiable synapses: A model of short and long term auto-associative memory. Proceedings of the Royal Society of London, B, $238,137-154$.

Gingrich, K. J., \& Byrne, J. H. (1985). Simulation of synaptic depression, posttetanic potentiation, and presynaptic facilitation of synaptic potentials from sensory neurons mediating gill-withdrawal reflex in Aplysia. Journal of Neurophysiology, 53, 652-669.

Gluck, M. A., \& Thompson, R. F. (1987). Modeling the neural substrates of associative learning and memory: A computational approach. Psychological Review, 94, 1-16.

Greenough, W. T., \& Bailey, C. H. (1988). The anatomy of a memory: Convergence of results across a diversity of tests. Trends in Neuroscience, 11, 142-147.

Hawkins, R. D., Clark, G. A., \& Kandel, E. R. (1987). Cell biological studies of learning in simple vertebrate and invertebrate systems. In F. Plum (Ed)., Handbook of physiology, Section 1: The nervous system, vol. 5: Higher functions of the brain, part 1, pp. 25-83. Bethesda, MD: American Physiological Society.

Hawkins, R. D., Kandel, E. R., \& Siegelbaum, S. A. (1993). Learning to modulate transmitter release: Themes and variations in synaptic plasticity. Annual Review of Neuroscience, 16, 625-665.

Herrera, A. A., Grinnell, A. D., \& Wolowske, B. (1985). Ultrastructural correlates of experimentally altered transmitter release efficacy in frog motor nerve terminals. Neuroscience, 16, 491-500.

Herrick, C. J. (1933). The amphibian forebrain: VIII. Cerebral hemispheres and pallial primordia. Journal of Comparative Neurology, 58, 737-759.

Hodgkin, A. L., \& Huxley, A. F. (1952). A quantitative description of membrane current and its application to conduction and excitation in nerve. Journal of Physiology (London), 117, 500-544.

Ingle, D. (1983). Brain mechanisms of visual localization by frogs and toads. In J.-P. Ewert, R. R. Capranica, \& D. Ingle (Eds.), Advances in vertebrate neuroethology. New York: Plenum.

Kohonen, T. (1989). Self-organization and associative memory (3rd ed.). New York: Springer-Verlag.

Lara, R., \& Arbib, M. A. (1985). A model of the neural mechanisms responsible for pattern recognition and stimulus specific habituation in toads. Biological Cybernetics, 51, 223-237.

Leaton, R. N. (1976). Long-term retention of the habituation of lick suppression 
and startle response produced by a single auditory stimulus. Journal of Experimental Psychology: Animal Behavioral Processes, 2, 248-259.

Leaton, R. N., \& Supple, W. F., Jr. (1991). Medial cerebellum and long-term habituation of acoustic startle in rats. Behavioral Neuroscience, 105, 804-816.

Llinás, R., \& Precht, W. (Eds.). (1976). Frog neurobiology. Berlin: Springer.

Lnenicka, G. A., Atwood, H. L., and Marin, L. (1986). Morphological transformation of synaptic terminals of a phasic motoneuron by long-term tonic stimulation. Journal of Neuroscience, 6, 2252-2258.

Magleby, K. L., \& Zengel, J. E. (1982). A quantitative description of stimulationinduced changes in transmitter release at the frog neuromuscular junction. Journal of General Physiology, 80, 613-638.

McFadden, P. N., \& Koshland, D. E., Jr. (1990). Parallel pathways for habituation in repetitively stimulated PC12 cells. Neuron, 4, 615-621.

Nagumo, J., Arimoto, S., \& Yoshizawa, S. (1962). An active pulse transmission line simulating nerve axon. Proceedings of the Institute of Radio Engineers, 50, 20612070.

Ögmen, H., \& Moussa, M. (1993). A neural model for nonassociative learning in a prototypical sensory-motor scheme: The landing reaction in flies. Biological Cybernetics, 68, 351-361.

Stanley, J. C. (1976). Computer simulation of a model of habituation. Nature, 261, 146-148.

Thompson, R. F. (1986). The neurobiology of learning and memory. Science, 233, 941-947.

Thompson, R. F., \& Spencer, W. A. (1966). Habituation: A model phenomenon for the study of neuronal substrates of behavior. Psychological Review, 73, 16-43.

Thorpe, W. H. (1956). Learning and instinct in animals. Cambridge, MA: Harvard University Press.

Tomsic, D., \& Maldonado, H. (1990). Central effect of morphine pretreatment on short- and long-term habituation to a danger stimulus in the crab Chasmagnathus. Pharmacology, Biochemistry, and Behavior, 36, 787-793.

Wang, D. L., \& Arbib, M. A. (1991a). How does the toad's visual system discriminate different worm-like stimuli? Biological Cybernetics, 64, 251-261.

Wang, D. L., \& Arbib, M. A. (1991b). Hierarchical dishabituation of visual discrimination in toads. In J.-A. Meyer \& S. Wilson (Eds.), Simulation of adaptive behavior: From animals to animats. Cambridge, MA: MIT Press.

Wang, D. L., \& Arbib, M. A. (1992). Modeling the dishabituation hierarchy: The role of the primordial hippocampus. Biological Cybernetics, 67, 535-544.

Wang, D. L., \& Ewert, J.-P. (1992). Configurational pattern discrimination responsible for dishabituation in common toads Bufo bufo (L.): Behavioral tests of the predictions of a neural model. Journal of Comparative Physiology, A, 170, 317-325.

Wang, D. L., \& Hsu, C. C. (1990). SLONN: A simulation language for modeling of neural networks. Simulation, 55, 69-83. 


\section{out the Authors}

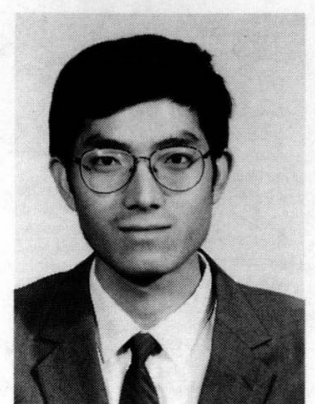

\section{DeLiang Wang}

DeLiang Wang is an assistant professor in the Department of Computer and Information Science and Center for Cognitive Science at Ohio State University, Columbus, $\mathrm{OH}$. He holds a bachelor of science degree (1983) and a master of science degree (1986) from Beijing University, Beijing, China, and a doctorate (1991) from the University of Southern California, Los Angeles, CA, all in computer science. His present research interests include temporal pattern processing, auditory and visual pattern perception, neural mechanisms of visuomotor coordination, neural network theories, and computational neuroscience. 